\title{
Dynamic evaluation of blood flow microcirculation by combined use of the laser Doppler flowmetry and high-speed videocapillaroscopy methods
}

Viktor Dremin ${ }^{1,2 *}$, Igor Kozlov ${ }^{1}$, Mikhail Volkov ${ }^{3}$, Nikita Margaryants ${ }^{3}$, Andrey Potemkin ${ }^{3}$, Evgeny Zherebtsov ${ }^{1,2}$, Andrey Dunaev ${ }^{1}$, and Igor Gurov ${ }^{3}$

*Corresponding Author: E-mail: dremin_viktor@mail.ru

${ }^{1}$ Research \& Development Center of Biomedical Photonics, Orel State University named after I.S. Turgenev, Komsomolskaya Str. 95, 302026, Orel, Russia

${ }^{2}$ Optoelectronics and Measurement Techniques unit, University of Oulu, Erkki KoisoKanttilankatu 3, 390014, Oulu, Finland

${ }^{3}$ ITMO University, Kronverksky Ave 49, 197101, St. Petersburg, Russia

Keywords: laser Doppler flowmetry, videocapillaroscopy, blood flow velocity oscillations, wavelet coherence, occlusion test, reverse blood flow

\section{Short title: V. Dremin et al.: Dynamic evaluation of blood flow microcirculation}

The dynamic light scattering methods are widely used in biomedical diagnostics involving evaluation of blood flow. However, there exist some difficulties in quantitative interpretation of backscattered light signals from the viewpoint of diagnostic information. This study considers the application of the high-speed videocapillaroscopy method that provides the direct measurement of the red blood cells velocity into a capillary. The videocapillaroscopy signal presents true oscillation nature of backscattered light caused by moving red blood cells. Thus, the videocapillaroscopy signal can be assigned as a reference one with respect to more complicated signals like in laser Doppler flowmetry. An essential correlation between blood flow velocity oscillations in a separate human capillary and the integral perfusion estimate obtained by the laser Doppler flowmetry method has been found. The observation of blood flow by the videocapillaroscopy method during upper arm occlusion has shown emergence of the reverse blood flow effect in capillaries that corresponds to the biological zero signal in the laser Doppler flowmetry. The reverse blood flow effect has to be taken into account in interpretation of laser Doppler flowmetry signals.

\section{Introduction}

This article has been accepted for publication and undergone full peer review but has not been through the copyediting, typesetting, pagination and proofreading process, which may lead to differences between this version and the Version of Record. Please cite this article as doi: $10.1002 / \mathrm{jbio} .201800317$ 
The dynamic light scattering (DLS) methods are widely used in biomedical diagnostics including evaluation of blood flow [1]. However, there exist some difficulties in the evaluation and quantitative interpretation of backscattered light signals from the viewpoint of diagnostic information. The well-known Laser Doppler Flowmetry (LDF) method [2] has been widely employed in many research applications. However, the high spatial variability in blood flow across living tissue limits the clinical utility of single point LDF measurements [3]. The full-field Laser Doppler Perfusion Imaging (LDPI) allows overcoming this limit, but does not provide high enough signal-to-noise ratio and, consequently, high evaluation speed that can be achieved in the Laser Speckle Contrast Imaging (LSCI) [3,4], but with limited resolution caused by spatial averaging. Diffusing-Wave Spectroscopy (DWS), also called diffuse correlation spectroscopy [5,6], allows studying the dynamics of scattered light under assumption of strong multiple scattering. However, this method is associated with the analytical solution of complex equations of the transfer theory, which leads to the complexity of interpreting the results. All these methods relate to well known approaches in studying blood microcirculation for early diagnosis of various diseases and monitoring the effectiveness of therapeutic impacts. However, the analysis and physiological interpretation of the blood flow oscillations remain an open problem for these methods. The blood flow fluctuations are often considered as a source of signal non-repeatability arising from the stochastic nature of red blood cells (RBCs) velocity fluctuations [7]. At the same time, local spectral decomposition of long-term perfusion records allows one to reveal the oscillatory components corresponding to the specific physiological mechanisms. Such local spectral decomposition can be provided particularly by the wavelet transformation of the LDF signals. Cardiac bands $(0.45-1.6 \mathrm{~Hz})$ and respiratory bands $(0.2-0.45 \mathrm{~Hz})$ provide information about the influence of heart rate and thorax movement on the peripheral blood flow. The myogenic mechanism of vascular tone regulation reflects the response of vascular smooth muscle cells to the transmural pressure. It is characterized by oscillations of blood flow at frequencies 
0.05-0.15 Hz. The neurogenic sympathetic vasomotor activity induces vessels walls movement with frequency $0.02-0.05 \mathrm{~Hz}$. Slow blood flow waves $(0.005-0.0095 \mathrm{~Hz}$ and 0.0095-0.02 Hz) indicates the vascular tone regulation due to the endothelium activity, both NO-dependent and independent. These mechanisms are considered in detail in Refs. [7-9]. Thus, useful diagnostic information is contained in various spectral bands, and time-frequency signal analysis is of high importance. However, there are still questions, for instance, whether the integral parameter of perfusion corresponds to a real change of blood flow velocity in capillaries. It is important to clarify the mechanism of the LDF signal origin involving direct evaluation of the RBCs velocity into capillaries.

Various recent studies have shown possibility of improving the quality of LDF measurements through a multimodal approach consisting in the combined use of several methods. LDF in combination with contact thermometry has improved the sensitivity and specificity of diagnosing patients with angiospastic violations in the vascular system [10]. The use of LDF and fluorescence spectroscopy allowed for the qualitative diagnosis of diabetes complications [11]. Due to the higher efficiency of multimodal approaches, we propose in the present study to use the videocapillaroscopy (VCS) method as a technique based on different physical principle with respect to LDF measurements that provides new and reliable results.

The VCS is based on high resolving registration and analysis of moving RBCs with evaluation of their velocity in a separate capillary by recording a video frames series representing a nail fold capillary blood flow. It extracts the parameters of capillary blood flow of individual capillaries as well as into a capillary net [12].

It should be noted that other methods can be used to determine the blood flow velocity. For instance, the Doppler Optical Coherence Tomography (DOCT) methods have been used for evaluation of the flow velocity in blood vessels [13]. However, conventional DOCT systems are unable to measure blood flow velocity in a wide field of view at all the points along a vessel length simultaneously. Moreover, conventional DOCT systems provide a lower spatial 
resolution (about $10 \mu \mathrm{m}$ ) in comparison with high-resolving nailfold VCS (up to $1 \mu \mathrm{m}$ ). Finally, the DOCT does not present a method of direct velocity measurement being based on the same Doppler effect as the LDF.

The Sidestream Dark Field imaging method is known as well. This technique allows for tracking the movement of individual blood cells with high resolution [14]. However, some technical limits inherent in this method make it inapplicable to observe the RBCs with high movement velocity, which is especially important in the study of blood flow, e.g., in the hyperemia stage $[15,16]$.

In the present research, we exploit a high-speed camera and advanced data processing algorithm that provide high accuracy of RBCs velocity measurements up to several $\mathrm{mm} / \mathrm{s}$ [12]. Moreover, the combination of LDF and high-speed VCS is motivated by the involvement two methods with different physical principles, so the results are independent of each other, and their comparison makes the results more reliable. It has been shown in previous studies that combined approach can be used to validate photoplethysmographic records [17]. A singlecapillary estimate of RBCs velocity may also be of particular interest for many applications of LDF measurements. The combined use of the LDF and VCS methods can resolve many doubts concerning the LDF method, for example, about the origin of LDF signal oscillations. The VCS method allows direct measurement of the RBCs velocity in a capillary thus presenting true oscillation nature of the signal caused by moving RBCs. Thus, this method can verify the data obtained by LDF devices, as well as substantiate interpretation of identified features of LDF signals. In this paper, the dynamics of perfusion in a distal finger phalanx and capillary blood flow (RBCs velocity) using the LDF and VCS methods, respectively, are considered.

\section{Material and Methods}

\subsection{Experimental setup}


The LDF method is based on the optoelectronic system configuration represented by the schematic diagram shown on the left in Figure 1.

The single-mode laser with $1064 \mathrm{~nm}$ wavelength was utilized as a radiation source. Optical fibers were used to deliver radiation to the skin surface and to collect backscattered light.

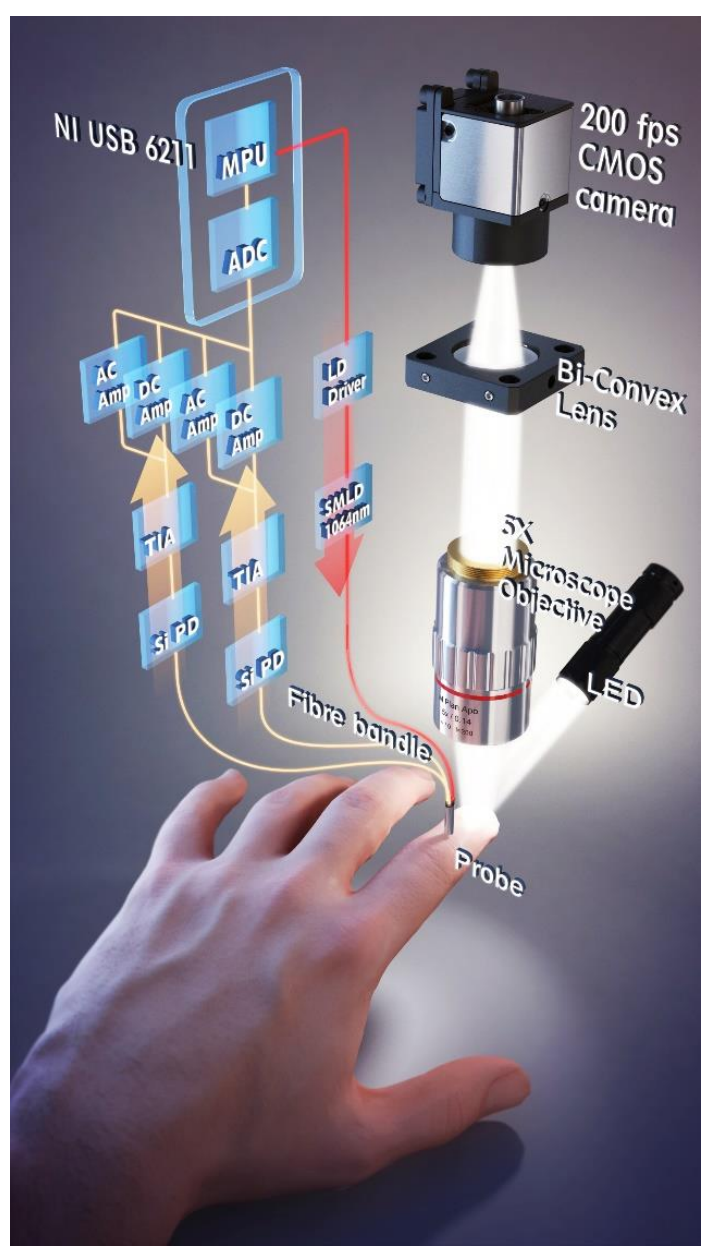

Figure 1. The overall scheme of the system. SMLD, single-mode laser diode; LD driver, laser diode driver; Si PD, silicon photodiode; TIA, transimpedance amplifier; AC Amp, AC signal amplifier; DC Amp, DC signal amplifier; ADC, analogue-to-digital converter; MPU, microprocessing unit.

Due to the technical limit of the LDF measurements within the nail bed area, the LDF signals were recorded at the middle phalanx of the same finger.

From the viewpoint of the light intensity distribution in a tissue, the sampling volume for the wavelength of $1064 \mathrm{~nm}$ depends less on the blood absorption and oxygen saturation, which 
reduce unwanted variability of the registered signal from measurement to measurement. Consequently, the assessment of perfusion parameter at the wavelength of $1064 \mathrm{~nm}$ characterizes mainly the velocity of scattering particles rather than the absorbing properties of living tissue.

The LDF probe sampling volume simulation based on the Monte Carlo technique [11] has shown that the diagnostic volume is about $1.8 \mathrm{~mm}^{3}$. In this case, the probe is sensitive to variations of blood flow in the papillary dermis and upper blood net plexus. The probe diagnostic volume covers the top part of the reticular dermis, which corresponds to the classical understanding of the sampling volume in the LDF method. Si-photodiodes were used to convert detected backscattered radiation into photocurrent. Then the signal was amplified by a custom electronic board. In this implementation of LDF setup, a differential circuit is used, which makes it possible to remove common-mode non-informative signal components for two channels. The analog-to-digital conversion was performed by the data acquisition board model NI USB 6211 (National Instruments, USA). Signal processing is conducted in the PC with NI LabVIEW environment.

The study of a nail fold capillary network was performed using the experimental VCS setup (see Figure 1 on the right) providing direct evaluation of the RBCs velocity. The nail fold area of the hand index finger was illuminated by a white LED. A $5^{\mathrm{x}}$ microscope objective with aperture 0.14 and a long-focus lens formed a capillary image on a photosensitive array. During the experiments, a high-speed CMOS camera with $200 \mathrm{fps}$ was acquiring the video data. The high-speed VCS technique enables to obtain detailed information about the RBCs velocity into a capillary as well as capillary net. The detailed description of algorithms for video frames processing is presented in Refs. [12,17,18].

\subsection{Correlation of perfusion and RBCs velocity in a basal state}


To study the general relationship of the signals in the basal state, the LDF signal and RBCs velocity in a single capillary were recorded in parallel for 14 minutes. The data was acquired at the sampling frequency of $250 \mathrm{~Hz}$ in VCS method and $20 \mathrm{~Hz}$ in LDF method. For the comparative data analysis, we have taken the VCS data at the time points corresponding to the LDF data samples.

The both LDF and VCS signals contain useful information about blood flow but in different form. It is important to evaluate the correspondence between the signals involving additional information provided by the VCS data to verify the LDF signal origin. Due to specific and local-variable properties of the signals mentioned above and inherent in blood flow study, it is reasonable to apply local spectral analysis implemented in the form of the continuous wavelet transform (CWT).

LDF and VCS signals were decomposed using CWT in the form:

$$
W(s, \tau)=\frac{1}{\sqrt{s}} \int_{-\infty}^{\infty} x(t) \psi^{*}\left(\frac{t-\tau}{s}\right) d t,
$$

where $x(t)$ is a target signal, $\tau$ is local time index, $s$ is scaling factor, * means complex conjugation. The Morlet wavelet defined in the form

$$
\psi(t)=e^{2 \pi i t} e^{-t^{2} / \sigma}
$$

was used with the decay parameter $\sigma=1$. This wavelet allows one to ensure sufficient timefrequency resolution and is well localized in the time domain.

The combined study of the VCS and LDF signals has to be conducted locally in time as well as at different frequencies. Such time-frequency comparison of two non-stationary signals can be provided involving the wavelet-based coherence. The wavelet transform coherence allows one to calculate the local correlation of two functions $x(t)$ and $y(t)$ with wavelet transforms $W^{x}(s, \tau)$ and $W^{y}(s, \tau)$ in the time-frequency domain [19-21]. This value is calculated in the form 


$$
R^{2}(s, \tau)=\frac{\left|H\left(s^{-1} W^{x y}(s, \tau)\right)\right|^{2}}{H\left(s^{-1}\left|W^{x}(s, \tau)\right|^{2}\right) \cdot H\left(s^{-1}\left|W^{y}(s, \tau)\right|^{2}\right)},
$$

where $R^{2}(s, \tau)$ ranges between 0 and $1, W^{x y}(s, \tau)=W^{x}(s, \tau) W^{y *}(s, \tau)$ is a cross-wavelet spectrum, $H$ is a smoothing operator. The smoothing in Eq. (3) is based on a weighted moving average in both the time and scale directions as described in detail by Torrence and Compo [22]. The smoothing assists to remove the singularities in wavelet power spectra and improves the reliability of calculations in Eq. (3).

The degree of correlation between the phases of the analyzed signals was also estimated from the viewpoint of the wavelet phase coherence [23].

The significance of the correlation is verified by constructing the surrogate data. For a surrogate generation we employed the method of amplitude-adjusted Fourier transform (AAFT). The AAFT produces surrogates having original values retained in the time series but rearranged in a way that largely preserves the spectrum while destroying temporal information [24]. We provided 100 pairs of surrogate data for each pair of experimental signal samples. Significance levels were calculated as the $95^{\text {th }}$ percentile of the surrogates.

Previously, the calculation of wavelet-based coherence was carried out to study the relationship among skin temperature, blood flow, tissue saturation, ECG signals, etc. This tool revealed significant relationships between skin temperature oscillations and peripheral blood flow [25,26], tissue saturation and peripheral blood flow [27], peripheral blood flow of contralateral skin sites [28].

For reliable statistics, one should include at least 10 cycles for each of the frequency components under investigation. We were conducting 14 min records. Therefore, reliable results can be obtained only for frequencies exceeding approximately $0.01 \mathrm{~Hz}$. 
Evaluation of the wavelet-based coherence reveals correspondence between the VCS and LDF data through their local spectra, i.e. locally in time and at different frequencies. Such correspondence has been clarified in our research. The results are discussed below.

\subsection{Correlation of perfusion and RBCs velocity with arterial occlusion}

To study the behavior of the two signals under conditions other than normal, an arterial occlusion test was used. The experiments included the following procedures: recording of background level (basal state) of perfusion and RBC velocity (30 s), occlusion test (90 s) and post-occlusion hyperemia $(10 \mathrm{~s})$. The occlusion test was performed according to the conventional procedure involving the application of a brachial arterial occlusion cuff with the pressure of 200-220 $\mathrm{mm} \mathrm{Hg}$.

\section{Results and Discussion}

\subsection{Correlation of perfusion and RBCs velocity in a basal state}

The LDF signal (perfusion) and RBC velocity variations are shown in Figure 2a,b, respectively. Figures 2c,d correspond to the CWT representation. Figures $2 \mathrm{c}, \mathrm{d}$ show the CWT scalogram charts represented as a gradient coloured map with the distribution of the wavelet power of the signal in the time-frequency domain. The dark regions relate the lowest power, while the yellow ones correspond to highest values.
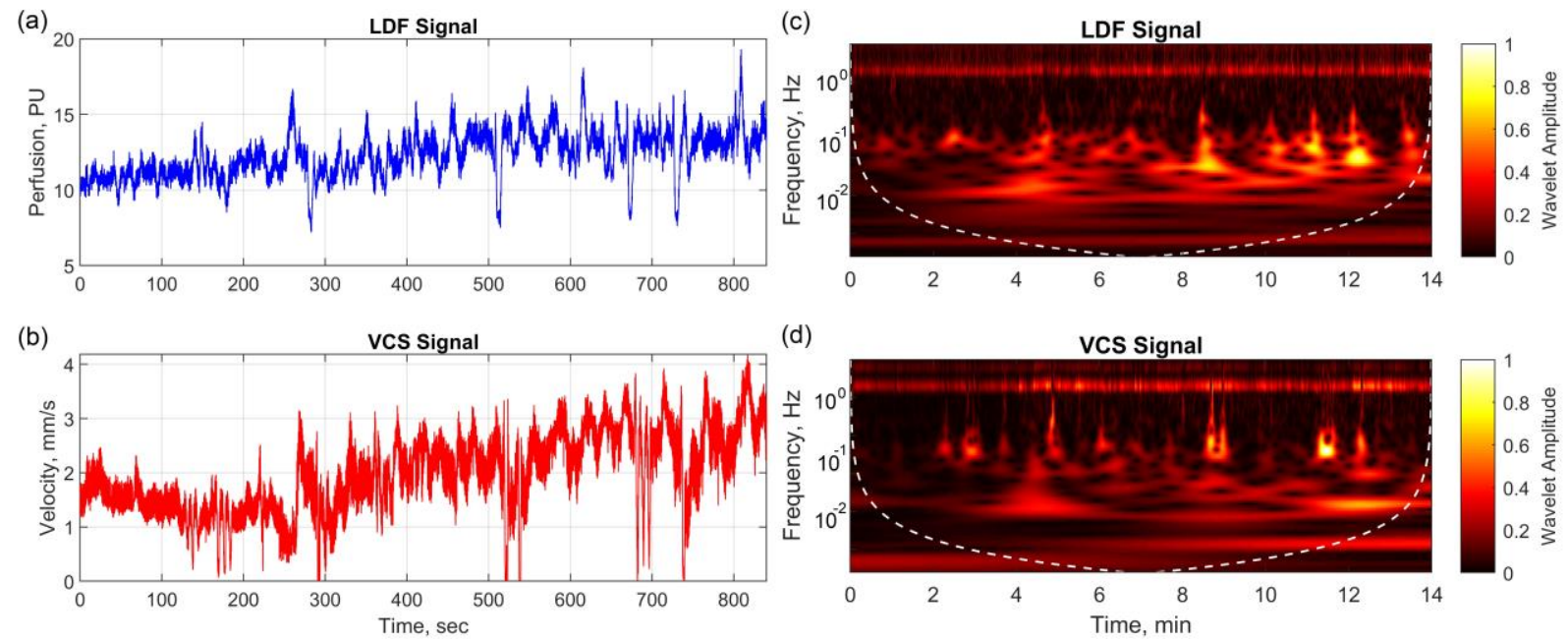
Figure 2. Experimental records of (a) LDF signal, (b) RBC velocity and (c, d) their CWT representation. White dash line shows the cone of influence where edge effects become significant at different frequencies.

As it is seen in Figure 2, the time changes of the raw signals evaluated by the two methods have a similarity, as well as their CWTs. Calculation of the wavelet transform coherence in correspondence with Eq. (3) confirms the similarity of signals and allows one to reveal their relationships and represent them in numerical form.
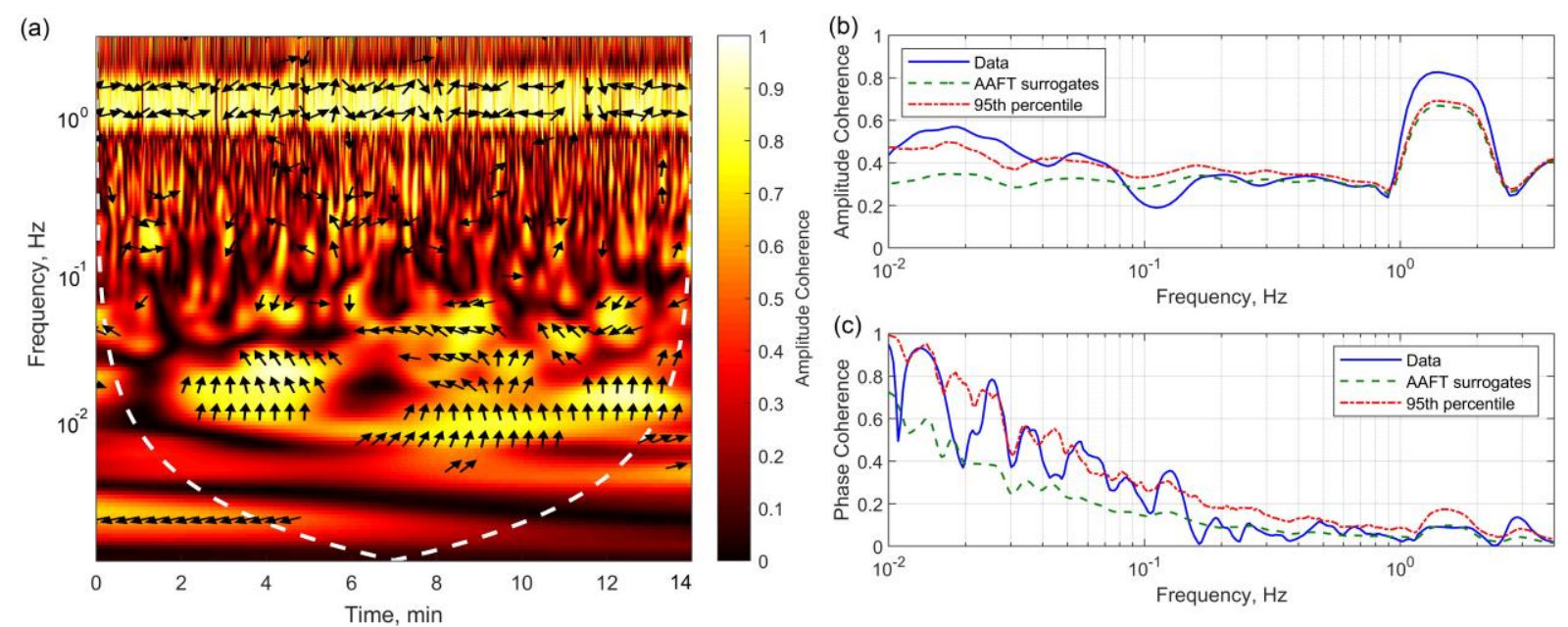

Figure 3. (a) Wavelet transform coherence between the LDF signal and RBCs velocity; (b) time-averaged wavelet coherence spectra and (c) wavelet phase coherence compared with the significance levels of the surrogate types shown, calculated as the $95^{\text {th }}$ percentile of 100 surrogates. Arrows show the phase lag of the LDF signal with respect to RBC velocity.

Figures 3a,b illustrate a region of significant coherence throughout the data collection period around 1-2 Hz. This is seen in the cardiac rhythms containing in the two signals. Additionally, the regions of significant coherence around $0.01-0.07 \mathrm{~Hz}$ are observed. In other frequency bands, e.g. respiratory bands, the coherence is not significant. This takes place due to small amplitude of pulsations in these bands and connection of the respiratory oscillations with the venous part of blood flow. For comparison, we show in Figure $3 \mathrm{~b}$ the mean coherence and the $95^{\text {th }}$ percentile calculated for 100 surrogates. It is worth noting that the areas of high 
coherence appearing outside or overlapping the cone of influence should be interpreted with caution.

Given the fact that the LDF signal is a superposition of blood flow velocity from different parts of the microvasculature with phase lag relative to each other, it is reasonable to assume the absence of phase coherence with the blood flow velocity in a single capillary. This assumption was confirmed by calculating the phase lag and phase coherence. In Figure 3a, the arrows show the phase lag of one signal relatively to another. The direction of the arrows correspond to the phase lag at the unit circle. The vertical arrow indicates $\pi / 2$ or quarter-cycle phase lag. The arrows pointing to the right or left show two in-phase or anti-phase signals, respectively. Figures 3a,c demonstrate the absence of significant phase coherence in almost entire frequency range.

It can be supposed that cardiac oscillations in the LDF signal are recorded from larger microvessels, i.e. arterioles. In normal conditions, the amplitude of pulse oscillations in capillaries is supposed to be low and hemodynamically insignificant. In this case, the presence of a capillary pulse may be an indicator of the course of a disease like microvascular pathology $[29,30]$. In our studies with healthy volunteers, we have shown that cardiac oscillation in the capillaries can make a significant contribution to the overall blood flow fluctuations.

The approach proposed has demonstrated the essential similarity of spectra oscillations in the isolated capillary and the integral estimation of the perfusion by the LDF method. This result demonstrates the deep connection of the LDF signal with objective physical characteristics of the skin blood microflow.

At the same time, the LDF method is often associated with the performance of various functional tests (breath holding, occlusive, pharmacological, etc.). The next section presents the comparative results of the observed perfusion and RBCs velocity in separate capillary during arterial occlusion test. 


\subsection{Correlation of perfusion and RBCs velocity during arterial occlusion}

For the first time the VCS method allowed for registration and quantification of such feature as «reverse» blood flow during the occlusion test. The video of this experiment is available online (see Movie S1). This effect identifies that the occlusion leads to a simultaneous reduction of blood flow in a single capillary from approximately $4 \mathrm{~mm} / \mathrm{s}$ to small negative values, and perfusion in the distal phalanx of the finger varies from 30 to 2 perfusion units. A subsequent gradual increase to 5 perfusion units with continued occlusion is caused by detectable reverse blood flow in the capillaries.

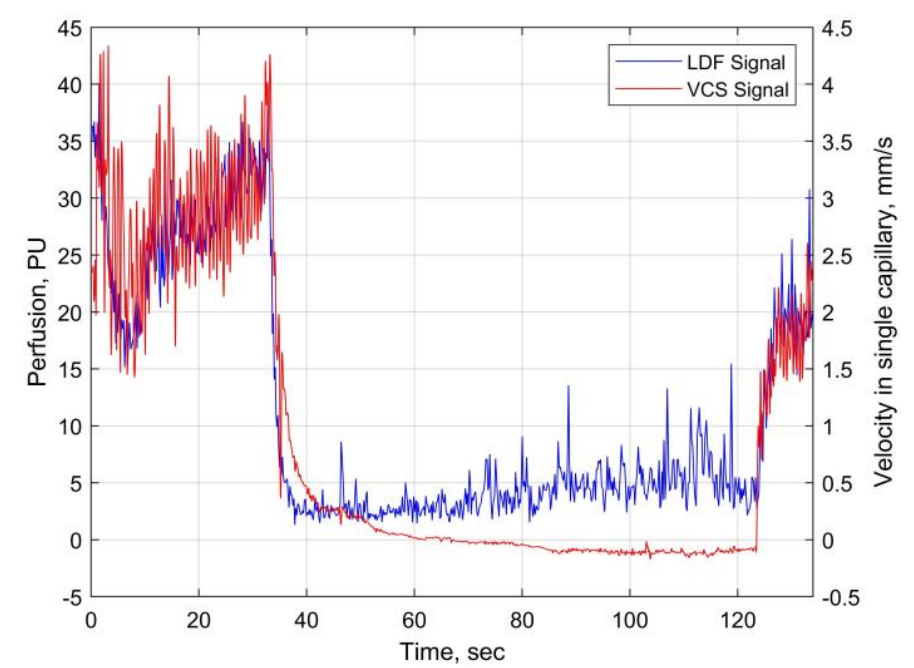

Figure 4. Experimental record of the LDF signal and RBCs velocity with reverse blood flow effect.

The absolute blood flow velocity in capillaries is increasing gradually from 0 to $0.2 \mathrm{~mm} / \mathrm{s}$ during the reverse blood flow effect registration (from $65 \mathrm{~s}$ to $120 \mathrm{~s}$ during the experiment). The finish of occlusion is characterized by a sharp increase in both the perfusion and RBCs velocity. The curves reflect a high degree of correspondence between the local variations of blood perfusion and RBCs velocity before and after the occlusion. However, there is no direct correlation within the occlusion period. It can be explained by the absence of cardiac activity 
influence on blood microcirculation during the occlusion and redistribution of pressure among microvessels.

The well-known problem of biological zero is studied for the cases when blood flow is blocked (e.g., during occlusion tests), but the signal is still detected [31,32]. Such a problem is considered when applying the LDF method or other methods based on registration of dynamic light scattering (in LDI, LSCI methods). It is believed in many publications that the thermal Brownian motion of particles (RBCs) is responsible for the presence of a signal [33]. However, some authors had shown in their studies non-Brownian nature of the RBCs movement after arterial occlusion. Meglinski et al. [34] have demonstrated that the presence of a directed blood flow after complete cessation of cardiac activity can be explained by the partially retained basal tonus of the vessels and leveling of the static pressure along the vascular bed. In the other research [35] the non-Brownian nature of blood flow is mentioned even after an hour of arterial occlusion of the mouse ear. The signal increase during arterial occlusion was also observed in our studies, but it correlated with the rate of the detected reverse blood flow. These facts indicate that the effect of biological zero should be described not only by thermal motion.

\section{Inclusion and exclusion criteria}

Ten healthy volunteers (6 males and 4 females, aged 21-40 years) were recruited to participate in the experiment. In total, over 30 capillaroscopy videos of various lengths were recorded. The results obtained were reliably repeated in various series of experiments with various volunteers.

This work represents an example of the long enough video records, which allowed analyzing the rhythms of blood flow up to the low frequency of $0.01 \mathrm{~Hz}$. In general, it can be noted that 
the data obtained for all volunteers demonstrated at least high coherence in the cardiac bands with short recordings. The phenomenon of reverse blood flow was detected in experiments involving all volunteers, but the effect was observed with different persons at different times after the onset of occlusion.

The study was approved by the ethics committee at Orel State University named after I.S. Turgenev (record of the meeting No. 10 of 16.11.2017) and was carried out in accordance with the principles outlined in the 2013 Declaration of Helsinki by the World Medical Association. The study protocol and its purpose were explained in detail to each volunteer and the informed consent was obtained from each person. The study included no volunteers with cardiovascular, bronchopulmonary, or neuroendocrine system disease comorbidities, no one with diseases of the gastrointestinal tract, liver, kidneys, blood, or with any other serious chronic diseases, which could have an impact on the results.

\section{Conclusion}

Analysis and interpretation of blood microcirculation present a complicated problem. The LDF is still one of the most attractive methods for that, whereas the difficulties of its application arise because of a lack of spatial visualization of the area of measurement and the inherently complex nature of microvascular flow. For this reason new modalities for blood flow evaluation assist in better understanding of microcirculation mechanisms. Particularly, the combined application of the LDF and VCS methods gives additional useful information to improve the data interpretation concerning with blood flow. It becomes possible to compare the changes in the perfusion in certain tissue volume in parallel with simultaneous evaluation of local RBCs velocity and its direction in a single capillary. The proposed approach with the application of wavelet transforms coherence to search time-localized coherent oscillatory behavior has demonstrated the essential similarity of spectra oscillations in the isolated capillary and the integral estimation of the perfusion by the LDF method. This study 
experimentally confirms the similarity between RBCs velocity in capillaries and blood perfusion of human skin evaluated by the LDF method and demonstrates the deep connection of the LDF signal origin with objective physical characteristics of the skin blood microflow.

An important and novel result of the paper is the observation that the presence of a LDF signal during occlusion (biological zero) can be caused not only by the Brownian motion of the RBCs, but also by the reverse blood flow. The current study adds an important element in our understanding of the characteristics of the blood flow and as such will be helpful in further research.

\section{Supporting Information}

Additional supporting information is available in the online version of this article on the publisher's website.

Movie S1. Experimental record of reverse blood flow during the arterial occlusion test. $65 \mathrm{~s}$ corresponds to the time at the beginning of the reverse blood flow.

\section{Acknowledgements}

This study was supported by the Russian Science Foundation under project No.18-15-00201 (collection and processing of experimental data by the CWT) and Ministry of Science and Higher Education of the Russian Federation under project No.8.2501.2017/4.6 (development of the experimental setup, processing and evaluation of the VCS experimental data). EZ kindly acknowledges for personal support from grant of Academy of Finland No.318281.

\section{References}

[1] V. V. Tuchin, Handbook of Coherent-Domain Optical Methods : Biomedical Diagnosis, Environmental Monitoring, and Materials Science (Springer, USA, 2013), pp. 1332.

[2] V. Rajan, B. Varghese, T. G. van Leeuwen, and W. Steenbergen, Lasers Med. Sci. 24, 269-283 (2009). 
[3] J. Allen, and K. Howell, Physiol. Meas. 35, R91-R141 (2014).

[4] S. M. Daly, and M. J. Leahy, J. Biophotonics 6, 217-255 (2013).

[5] Z. Fahimi, F. J. Aangenendt, P. Voudouris, J. Mattsson, and H. M. Wyss, Phys. Rev. E 96, 62611 (2017).

[6] M. Ninck, M. Untenberger, and T. Gisler, Biomed. Opt. Express 1, 1502-1513 (2010).

[7] G. Lancaster, A. Stefanovska, M. Pesce, G. Marco Vezzoni, B. Loggini, R. Pingitore, F. Ghiara, P. Barachini, G. Cervadoro, M. Romanelli, and M. Rossi, Sci. Rep. 5, 12825 (2015).

[8] H. D. Kvernmo, A. Stefanovska, K. A. Kirkeboen, and K. Kvernebo, Microvasc. Res. 57, 298-309 (1999).

[9] P. Kvandal, S. A. Landsverk, A. Bernjak, A. Stefanovska, H. D. Kvernmo, and K. A. Kirkebøen, Microvasc. Res. 72, 120-127 (2006).

[10] E. A. Zherebtsov, A. I. Zherebtsova, A. Doronin, A. V Dunaev, K. V Podmasteryev, A. Bykov, and I. Meglinski, J. Biomed. Opt. 22, 40502 (2017).

[11] V. V. Dremin, E. A. Zherebtsov, V. V. Sidorov, A. I. Krupatkin, I. N. Makovik, A. I. Zherebtsova, E. V. Zharkikh, E. V. Potapova, A. V. Dunaev, A. A. Doronin, A. V. Bykov, I. E. Rafailov, K. S. Litvinova, S. G. Sokolovski, and E. U. Rafailov, J. Biomed. Opt. 22, (2017). [12] I. Gurov, M. Volkov, N. Margaryants, A. Pimenov, and A. Potemkin, Opt. Lasers Eng. 104, 244-251 (2018).

[13] M. Bonesi, D. Churmakov, and I. Meglinski, Meas. Sci. Technol. 18, 3279-3286 (2007).

[14] P. T. Goedhart, M. Khalilzada, R. Bezemer, J. Merza, and C. Ince, Opt. Express 15, $15101(2007)$.

[15] M. Sorelli, L. Bocchi, and C. Ince, in: 37th Annu. Int. Conf. IEEE Eng. Med. Biol. Soc., 7378-7381 (2015).

[16] M. Sorelli, C. Ince, and L. Bocchi, Physiol. Meas. 38, 358-373 (2017).

[17] M. V. Volkov, N. B. Margaryants, A. V. Potemkin, M. A. Volynsky, I. P. Gurov, O. V Mamontov, and A. A. Kamshilin, Sci. Rep. 7, 13298 (2017). 
[18] M. V. Volkov, D. A. Kostrova, N. B. Margaryants, I. P. Gurov, N. P. Erofeev, V. V. Dremin, E. V. Zharkikh, E. A. Zherebtsov, I. O. Kozlov, and A. V. Dunaev, in: Proc. SPIE. 10336, 1033607 (2017).

[19] A. Grinsted, J. C. Moore, and S. Jevrejeva, Nonlinear Process. Geophys. 11, 561-566 (2004).

[20] C. Chang, and G. H. Glover, Neuroimage 50, 81-98 (2010).

[21] X. Cui, D. M. Bryant, and A. L. Reiss, Neuroimage 59, 2430-2437 (2012).

[22] C. Torrence, and G. P. Compo, Bull. Am. Meteorol. Soc. 79, 61-78 (1998).

[23] A. Bandrivskyy, A. Bernjak, P. McClintock, and A. Stefanovska, Cardiovasc. Eng. An Int. J. 4, 89-93 (2004).

[24] G. Lancaster, D. Iatsenko, A. Pidde, V. Ticcinelli, and A. Stefanovska, Phys. Rep. 748, $1-60(2018)$.

[25] P. Frick, I. Mizeva, and S. Podtaev, Biomed. Signal Process. Control 21, 1-7 (2015).

[26] L. W. Sheppard, V. Vuksanović, P. V. E. McClintock, and A. Stefanovska, Phys. Med. Biol. 56, 3583-3601 (2011).

[27] A. Bernjak, A. Stefanovska, P. V. E. McClintock, P. J. Owen-Lynch, and P. B. M. Clarkson, Fluct. Noise Lett. 11, 1240013 (2012).

[28] A. V. Tankanag, A. A. Grinevich, T. V. Kirilina, G. V. Krasnikov, G. M. Piskunova, and N. K. Chemeris, Microvasc. Res. 95, 53-59 (2014).

[29] A. C. Guyton, Textbook of Medical Physiology (Elsevier Saunders, USA, 2006), pp. 1152.

[30] C. G. Caro, T. J. Pedley, R. C. Schroter, W. A. Seed, and K. H. Parker, The Mechanics of the Circulation, (Cambridge University Press, UK, 2011), pp. 524.

[31] C. Millet, M. Roustit, S. Blaise, and J. L. Cracowski, Microvasc. Res. 82, 147-151 (2011).

[32] A. Humeau-Heurtier, P. Abraham, and G. Mahe, IEEE Trans. Med. Imaging 32, 2311- 
$2321(2013)$.

[33] T. Binzoni, T. S. Leung, M. L. Seghier, and D. T. Delpy, Phys. Med. Biol. 49, 5445-58 (2004).

[34] I. V. Meglinski, V. V. Kalchenko, Y. L. Kuznetsov, B. I. Kuznik, and V. V. Tuchin, Dokl. Phys. 58, 323-326 (2013).

[35] V. Kalchenko, A. Brill, M. Bayewitch, I. Fine, V. Zharov, E. Galanzha, V. Tuchin, and A. Harmelin, J. Biomed. Opt. 12, 052002 (2007).

Figure 1. The overall scheme of the system. SMLD, single-mode laser diode; LD driver, laser diode driver; Si PD, silicon photodiode; TIA, transimpedance amplifier; AC Amp, AC signal amplifier; DC Amp, DC signal amplifier; ADC, analogue-to-digital converter; MPU, microprocessing unit.

Figure 2. Experimental records of (a) LDF signal, (b) RBC velocity and (c, d) their CWT representation. White dash line shows the cone of influence where edge effects become significant at different frequencies.

Figure 3. (a) Wavelet transform coherence between the LDF signal and RBCs velocity; (b) time-averaged wavelet coherence spectra and (c) wavelet phase coherence compared with the 
significance levels of the surrogate types shown, calculated as the 95th percentile of 100 surrogates. Arrows show the phase lag of the LDF signal with respect to RBC velocity.

Figure 4. Experimental record of the LDF signal and RBCs velocity with reverse blood flow effect.

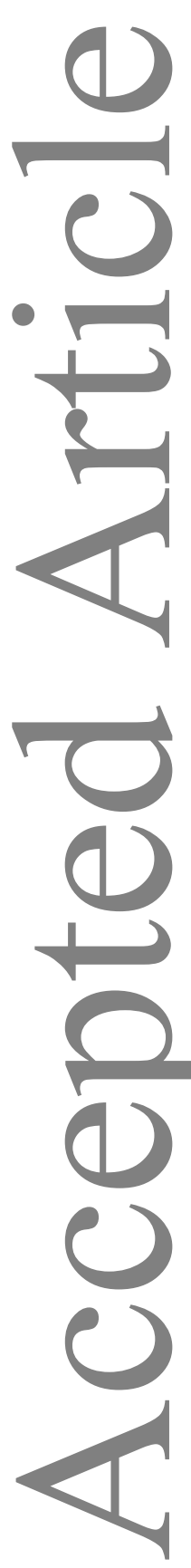

\section{Graphical Abstract}

The high-speed videocapillaroscopy method provides unique information about the capillary net. This study empirically confirms the similarity between red blood cells velocity into capillaries and blood perfusion evaluated by the laser Doppler flowmetry method, thus demonstrating the deep connection of the laser Doppler flowmetry signal origin with objective physical characteristics of the skin blood microflow. 
